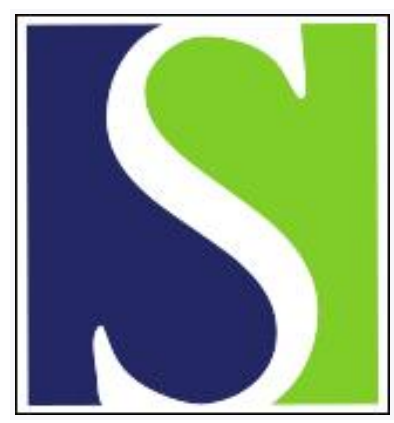

Scand J Work Environ Health 2018;44(2):202-211

https://doi.org/10.5271/sjweh.3688

Published online: 02 Nov 2017, Issue date: 01 Mar 2018

Objectively measured occupational and leisure-time physical activity: cross-sectional associations with sleep problems

by Skarpsno ES, Mork PJ, Nilsen TIL, Jørgensen MB, Holtermann A

This study provides new knowledge about the association between objectively measured occupational physical activity (OPA), lesiure-time physical activity (LTPA) and sleep problems. Our analyses showed that OPA is positively and dose-dependently associated with insomnia symptoms while LTPA is inversely associated with non-restorative sleep. These findings underscore the domain-dependent association between physical activity and health-related outcomes.

Affiliation: Department of Public Health and Nursing, Norwegian University of Science and Technology (NTNU), 7491 Trondheim, Norway. eivind.s.skarpsno@ntnu.no

Refers to the following text of the Journal: 2016;42(4):291-298

Key terms: accelerometer; DPhacto; insomnia; leisure-time physical activity; non-restorative sleep; occupational physical activity; physical activity; physical activity health paradox; sleep; sleep problem; technical measurement; worker

This article in PubMed: www.ncbi.nlm.nih.gov/pubmed/29094169

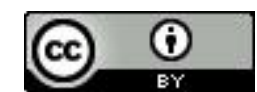




\title{
Objectively measured occupational and leisure-time physical activity: cross-sectional associations with sleep problems
}

\author{
by Eivind Schjelderup Skarpsno, MSc, , 2 Paul Jarle Mork, PhD, 1, Tom Ivar Lund Nilsen, PhD, 1 \\ Marie Birk Jørgensen, PhD, ${ }^{3}$ Andreas Holtermann, $P h D^{3,4}$
}

\begin{abstract}
Skarpsno ES, Mork PJ, Nilsen TIL, Jørgensen MB, Holtermann A. Objectively measured occupational and leisure-time physical activity: cross-sectional associations with sleep problems. Scand J Work Environ Health. 2018;44(2):202-211. doi:10.5271/sjweh.3688
\end{abstract}

\begin{abstract}
Objectives This study aimed to investigate (i) the associations between occupational physical activity (OPA) and leisure-time physical activity (LTPA) with insomnia symptoms and non-restorative sleep and (ii) the joint associations between OPA and LTPA with insomnia symptoms and non-restorative sleep, respectively.

Methods Data were drawn from a cross-sectional study including 650 workers in the Danish PHysical ACTivity cohort with Objective measurements (DPhacto). OPA and LTPA were measured with accelerometers on the thigh and upper back for up to six consecutive days and subsequently divided into quartiles of "very low", "low", "medium" and "high" activity. We used logistic regression to calculate odds ratios (OR) with 95\% confidence intervals (CI) for insomnia symptoms and non-restorative sleep associated with OPA and LTPA.
\end{abstract}

Results A 10\% increase in OPA was associated with a higher prevalence of insomnia symptoms (OR 1.39, 95\% CI 1.03-1.89) but not with the prevalence of non-restorative sleep (OR 0.93, 95\% CI 0.71-1.21). On the other hand, a $10 \%$ increase in LTPA was associated with a lower prevalence of non-restorative sleep (OR 0.51, 95\% CI 0.28-0.92). Although no significant additive interaction was found, analyses of the joint association of OPA and LTPA showed that people with high OPA and low LTPA had an OR of 2.07 (95\% CI 1.01-4.24) for insomnia symptoms, compared to those with low OPA and high LTPA, whereas people with high levels of both OPA and LTPA had an OR of 1.47 (95\% CI 0.73-2.96).

Conclusions While LTPA was associated with lower prevalence of sleep problems, OPA was associated with higher prevalence of insomnia symptoms. A combination of high OPA and low LTPA were more strongly associated with insomnia symptoms compared to a combination of low OPA and high LTPA.

Key terms accelerometer; DPhacto; insomnia; non-restorative sleep; occupational physical activity; physical activity health paradox; technical measurement; worker.

Sleep problems and insomnia are common in the general population (1-2), and associated with increased risk of cardiovascular disease, type 2 diabetes, hypertension, widespread chronic pain, and mental disorders (3-8). Both observational (9-11) and intervention (12, 13) studies report positive effects of physical activity on sleep, and physical activity is recommended as a pertinent non-pharmacological treatment of common sleep disorders (14). Physically active people report less problems falling asleep, less nocturnal awakenings and less daytime sleepiness (15-17). Furthermore, physical activity is associated with improvements in the feeling of sleep being restorative (18), probably because of a more favorable sleep architecture (14) with less awakenings (18). However, this research is almost exclusively based on studies of leisure-time physical activity (LTPA). Moreover, although emerging evidence suggest that non-restorative sleep can occur without other insomnia symptoms (19), few studies have investigated these symptoms separately.

For a large fraction of the population, the main domain for physical activity is at work (20). In contrast

1 Department of Public Health and Nursing, Norwegian University of Science and Technology (NTNU), Trondheim, Norway.

2 Liaison Committee between Central Norway Regional Health Authority and NTNU, Trondheim, Norway

3 National Research Centre for the Working Environment, Copenhagen, Denmark.

4 Department of Sports Science and Clinical Biomechanics, University of Southern Denmark, Odense, Denmark.

Correspondence to: Eivind Schjelderup Skarpsno, Department of Public Health and Nursing, Norwegian University of Science and Technology (NTNU), 7491 Trondheim, Norway. [E-mail: eivind.s.skarpsno@ntnu.no] 
to LTPA, occupational physical activity (OPA) is often characterized by constrained activities related to specific work tasks and productivity with little opportunity to rest at one's convenience (21). Accordingly, recent studies have shown that high OPA may have negative health consequences (22-24). The contrasting health effects of LTPA and OPA therefore indicate that the impact of physical activity on health is dependent on its domain (21). Because the underlying mechanism behind the contrasting effects of OPA and LTPA on health is still unresolved, it has been termed "the physical activity health paradox" (25).

Few studies have investigated the association between OPA and sleep problems. One study found that physically strenuous work was associated with disturbed sleep (26), while a recent study observed that moderateto-high OPA was associated with reduced sleep duration and sleep quality (27). However, the assessment of OPA in these studies was based on self-report that is subject to misclassification and could give biased associations $(28,29)$. Thus, the objective of the current study was to investigate (i) the associations between objectively measured OPA and LTPA with insomnia symptoms and non-restorative sleep, and (ii) the joint associations between OPA and LTPA with insomnia symptoms and non-restorative sleep, respectively.

\section{Methods}

\section{Study population and design}

This study is based on data from the Danish PHysical ACTivity cohort with Objective measurements (DPhacto). Data were collected from spring 2012 until spring 2014 from 15 workplaces located in different regions all over Denmark from three sectors (ie, manufacturing, cleaning, and transportation) with varying physical work demands. Detailed information about the DPhacto study is described elsewhere (30).

Participants were mainly blue-collar workers, but some of their colleagues in administrative and management positions (defined as white-collar workers) also participated. A total of 2107 workers were invited and, of these, 1119 workers consented to participate. Inclusion criteria were $\geq 20$ working hours per week and age 18-67 years. Exclusion criteria were pregnancy, having fever on the day of testing, band aid allergy, slipped disc, and current and/or past illness restricting the worker from participating in the measurements of physical activity. Objective measurements of OPA and LTPA were then obtained from 755 workers. Of these, 57 workers with missing accelerometer recordings at work and/or during leisure time were excluded. Of the remaining 698 workers, we excluded those missing information on alcohol consumption (28), body height and/or weight (11), type of work (4), and smoking (31). Thus, the analysis was based on 650 workers (294 women and 356 men).

The Ethics Committee for the Capital Region of Denmark approved the study, which was conducted in accordance with the Declaration of Helsinki. All workers who participated in the study signed an informed consent.

\section{Procedure}

All workers were invited to information meetings where the objectives and procedures of the study were explained in detail. The participants were asked to wear accelerometers for continuous measurement of physical activity during the entire day and night for up to six consecutive days, including at least two working and two leisure days. Research staff visited the workplace on the first and the last day of measurements. Measurements of height and weight were performed on the same day as mounting of the accelerometers for objective measurements of physical activity. The workers also completed a short computer-based questionnaire concerning age, sex, education, working hours, diet, smoking, alcohol consumption, physical activity, self-reported health, work ability, psychosocial work environment, and musculoskeletal pain. After the participants had completed all days of physical activity measurements, the workers returned a diary with information about working days, working hours, days off work, and periods without wearing the measuring devices.

\section{Objective measurements of physical activity}

Physical activity was recorded with two accelerometers (Actigraph GT3X+, Actigraph LLC, Pensacola, FL, USA) attached on the thigh and the upper back. Actigraph is a compact water-resistant device $(19 \times 34 \times 45$ $\mathrm{mm}$, weight $19 \mathrm{~g}$ ), which measures tri-axial acceleration with a frequency of $30 \mathrm{~Hz}$, a dynamic range of $\pm 6 \mathrm{G}$ (1 $\mathrm{G}=9.81 \mathrm{~m} / \mathrm{s}^{2}$ ), and a precision of $12 \mathrm{bit}$. The accelerometers were first initialized using the Actilife software version 5.5 (ActiGraph LLC, Pensacola, FL, USA). Data were processed off-line using the Acti4 software (31). The accelerometers were fixed by tape (3M, HairSet, double-sided adhesive tape, and Fixomull, BSN medical). A detailed description of the placement of the accelerometers has been reported elsewhere (31).

Non-wear periods were automatically identified and excluded from the analysis. The following criteria for detection of a non-wear period were used: (i) all periods $>60$ minutes with zero acceleration counts of the accelerometer, (ii) if the participants reported non-wear time, or (iii) artefacts and/or missing data were detected 
by visual inspection. Periods $<10$ minutes without any movement were not regarded as non-wear periods (31).

Different types of physical activity were detected from the processed accelerometer signals (32). The total time (hours/day) with physical activity, ie, walking, running, cycling and walking stairs was summed up separately during work and leisure time. OPA was then expressed as percentage of total time at work, and LTPA was expressed as percentage of total time during leisure time. Total physical activity was expressed as percentage of the total time by adding OPA and LTPA. OPA, LTPA, and total physical activity were then divided into quartiles to obtain four exposure groups: "very low", "low", "medium", and "high" [cut offs OPA: 9.9\% (49 minutes), $16.4 \%$ (78 minutes), $22.0 \%$ (102 minutes); cut offs LTPA: $7.2 \%$ (38 minutes), 9.7\% (52 minutes), 13.2\% (70 minutes), cut offs total physical activity: $10.0 \%, 12.8 \%$, $15.9 \%$ ]. The mean percentage time with OPA was $7.0 \%$ (34.9 minutes/day) for the very low group, $13.4 \%$ (64.2 minutes/day) for the low group, $19.2 \%$ (91.3 minutes/day) for the medium group, and $27.2 \%$ (121.2 minutes/day) for the high group. The corresponding mean percentage time for LTPA was $5.5 \%$ (30.3 minutes/day) for the very low group, $8.4 \%$ (46.1 minutes/day) for the low group, $11.3 \%$ (61.2 minutes/day) for the medium group, and 16.5\% (88.7 minutes/day) for the high group. In the analyses of joint associations, OPA and LTPA were dichotomized with the "very low" and "low" groups classified as "low", and the "medium" and "high" groups classified as "high". This information was then used to obtain a variable with four combined categories: (i) high LTPA and low OPA, (ii) high LTPA and high OPA, (iii) low LTPA and low OPA, and (iv) low LTPA and high OPA.

\section{Insomnia symptoms and non-restorative sleep}

Insomnia symptoms was assessed by two questions: "How often during the last month did you: 1) have difficulties falling asleep at night? and 2) wake up too early and couldn't get back to sleep?". The response options were: "never", "rarely", "sometimes", "often", and "always" on both questions. Participants answering "often" or "always" on one or both questions were considered to have symptoms of insomnia.

The frequency of experiencing non-restorative sleep was assessed by the question: "In the last month, how often have you felt that you were not rested when you woke up in the morning?". The response options were: "never", "rarely", "sometimes", "often", and "always". Participants answering "often" or "always" were considered to experience non-restorative sleep.

\section{Assessment of covariates}

Age was determined from the worker's Danish civil registration number (CPR-number). Body mass index $\left(\mathrm{BMI}, \mathrm{kg} / \mathrm{m}^{2}\right.$ ) was calculated using objectively measured height $(\mathrm{m})$ and body mass $(\mathrm{kg})$. Smoking was determined from the question: "Do you smoke?" with four response options: "yes, daily", "yes, sometimes", "I used to smoke, but not anymore", and "I have never smoked". The response options were further categorized into two groups: "yes" (yes, daily and yes, sometimes), and "no" (used to smoke, but not anymore, and I have never smoked). Alcohol consumption was determined from the question: "Do you drink alcohol?", if yes, the participants were asked to answer "How many units did you drink last week?". The answers were then categorized into tertiles (low, medium, high). Medication for depression was assessed by two questions: "Have you in the last three months been taking prescription medication?", with response options: "yes" and "no". If "yes", the participants were asked about type of medication, with anti-depressives as a response option. Based on the participant's workplace, we categorized workers into three sectors: cleaning, manufacturing, and transportation. Intensity and extent of musculoskeletal pain was assessed by the question: "During the last 3 months, what have been your worst pain in the following body regions on a scale from 0-10?". Body regions included "neck/shoulders", "elbows", "wrists/hands", "low back", "hips", "knees", and "ankles/feet". Based on these initial answers on pain, we constructed a new variable using number of pain sites and pain intensity to categorize participants into four strata: "no pain", "light pain", "1-2 pain sites", and " $\geq 3$ pain sites". Participants were categorized into "no pain" if they answered $\leq 2$ on pain intensity for all pain sites, "light pain" if they answered $\leq 5$ on intensity for all pain sites, " $1-2$ pain sites" if they answered $\geq 6$ on intensity for $1-2$ pain sites, and " $\geq 3$ pain sites" if they answered $\geq 6$ on intensity for $\geq 3$ pain sites. Shift work was assessed by the question: "At which time of the day do you usually work in your main occupation?", with the following response options: "fixed day work", "night, varying working hours with night", and "other". Number of working hours per week was assessed by the following question: "How many hours per week do you work in your main occupation?".

\section{Statistical analysis}

Logistic regression was used to calculate odds ratios (OR) for insomnia symptoms and non-restorative sleep associations with categories of OPA, LTPA and total physical activity. The precision of the associations was assessed by $95 \%$ confidence intervals (CI). Participants with "low", "medium" and "high" OPA or LTPA respectively were compared with the reference group of participants with "very low" OPA or LTPA, respectively. Trend tests across categories of OPA and LTPA were 
calculated by treating the categories as ordinal variables in the regression model. We also analyzed the data using continuous OPA and LTPA variables (ie, OR per 10\% difference). Moreover, we estimated the joint associations of OPA and LTPA with insomnia symptoms and non-restorative sleep. Participants with the combination of low OPA and high LTPA served as the reference group in this analysis. The multi-adjusted model was adjusted for sex, age (continuous), BMI (continuous), use of anti-depressives (yes, no), smoking (yes, no), shift work (yes, no), alcohol consumption (low, medium, high), musculoskeletal pain (no pain, light pain, 1-2 pain sites, and $\geq 3$ pain sites) and work type (cleaning, manufacturing, transportation).

Potential effect modification between the variables was assessed as departure from additive effects. Interaction on an additive scale was calculated as the relative excess risk due to interaction (RERI), but modified to reflect our use of OR: RERI $=\mathrm{OR}_{\text {low LTPA and high OPA }}-\mathrm{OR}_{\text {high }}$ LTPA and high OPA $-\mathrm{OR}_{\text {low LTPA and low OPA }}+1$ (33), ie, RERI $>0$ indicates a synergistic association beyond an additive association.

To test the robustness of the results, we conducted supplementary analyses (data not shown) where we investigated whether the same associations remained with another division of OPA and LTPA, and with exclusion of possible risk factors of sleep problems. We therefore conducted a sensitivity analysis using tertiles for OPA and LTPA. Moreover, night work and working hours are two known risk factors of sleep problems
$(34,35)$, and two sensitivity analyses were therefore conducted (i) excluding 61 workers with night shifts, and (ii) including working hours as a covariate in the multi-adjusted model.

All statistical analyses were performed using Stata for Windows, version 13.1 (StataCorp LP, College Station, TX, USA).

\section{Results}

\section{Characteristics of the study population}

Table 1 presents the characteristics of the study population stratified by quartiles of OPA. Of the 650 workers who participated in the study, 92 workers $(14.1 \%)$ reported insomnia symptoms, while $112(17.4 \%)$ reported non-restorative sleep. OPA consisted of $95.3 \%$ (74 minutes) walking, $4.0 \%$ ( 3 minutes) walking stairs, $0.3 \%$ (14 seconds) running, and $0.4 \%$ (19 seconds) cycling, whereas LTPA consisted of $85.6 \%$ (48 minutes) walking, $5.1 \%$ (3 minutes) walking stairs, $2.6 \%$ ( 1 minute 30 seconds) running, and $6.7 \%$ (4 minutes) cycling. High OPA (medium and high quartiles of OPA) consisted of 77 minutes walking (64 min fast walking) and $<1$ minutes of running/cycling, whereas high LTPA (medium and high quartiles of LTPA) consisted of 62 minutes walking (50 minutes fast walking), 3 minutes running, and 7 minutes cycling. Moreover, the remain-

Table 1. Characteristics of the study population. [SD=standard deviation; LTPA=leisure-time physical activity.]

\begin{tabular}{|c|c|c|c|c|c|c|c|c|c|c|c|c|c|c|c|c|c|c|c|c|}
\hline & \multicolumn{20}{|c|}{ Quartiles of occupational physical activity (OPA) } \\
\hline & \multicolumn{4}{|c|}{ Very low } & \multicolumn{4}{|c|}{ Low } & \multicolumn{4}{|c|}{ Medium } & \multicolumn{4}{|c|}{ High } & \multicolumn{4}{|c|}{ Total } \\
\hline & $\mathrm{N}$ & $\%$ & Mean & $\mathrm{SD}$ & $\mathrm{N}$ & $\%$ & Mean & SD & $\mathrm{N}$ & $\%$ & Mean & SD & $\mathrm{N}$ & $\%$ & Mean & SD & $\mathrm{N}$ & $\%$ & Mean & SD \\
\hline $\mathrm{N}$ & 167 & 26 & & & 166 & 25.7 & & & 162 & 25.5 & & & 155 & 23.8 & & & 650 & & & \\
\hline Females & 86 & 51.5 & & & 55 & 33.1 & & & 69 & 42.6 & & & 84 & 54.2 & & & 369 & 45.2 & & \\
\hline Smokers & 39 & 23.3 & & & 40 & 24.1 & & & 53 & 32.7 & & & 47 & 30.3 & & & 179 & 27.5 & & \\
\hline Shift work & 9 & 5.4 & & & 18 & 10.8 & & & 20 & 12.0 & & & 18 & 11.6 & & & 65 & 10.0 & & \\
\hline Insomnia symptoms & 16 & 9.6 & & & 20 & 12.0 & & & 27 & 16.7 & & & 29 & 18.4 & & & 92 & 14.1 & & \\
\hline Non-restorative sleep & 29 & 17.4 & & & 23 & 13.8 & & & 36 & 22.2 & & & 24 & 15.2 & & & 112 & 17.4 & & \\
\hline White-collar workers & 77 & 46 & & & 50 & 31 & & & 34 & 21 & & & 25 & 17 & & & 187 & 29 & & \\
\hline \multicolumn{21}{|l|}{ Sector } \\
\hline Cleaning & 5 & 3.0 & & & 11 & 6.6 & & & 36 & 22.2 & & & 62 & 39.2 & & & 114 & 17.4 & & \\
\hline Manufacturing & 154 & 92.2 & & & 143 & 85.6 & & & 117 & 72.2 & & & 77 & 48.7 & & & 491 & 75.1 & & \\
\hline Transportation & 8 & 4.8 & & & 13 & 7.8 & & & 9 & 5.6 & & & 19 & 12.0 & & & 49 & 7.5 & & \\
\hline Age & & & 45.5 & 8.7 & & & 45.0 & 9.5 & & & 44.8 & 10.2 & & & 44.9 & 10.8 & & & 45.1 & 9.8 \\
\hline Body mass index & & & 26.5 & 4.6 & & & 26.9 & 3.9 & & & 27.5 & 4.9 & & & 27.9 & 5.1 & & & 27.2 & 4.7 \\
\hline Alcohol consumption (units/week) & & & 4.6 & 5.7 & & & 5.0 & 6.1 & & & 4.4 & 6.3 & & & 4.2 & 6.2 & & & 4.5 & 6.1 \\
\hline Valid days of measurements & & & 2.2 & 0.8 & & & 2.2 & 0.8 & & & 2.0 & 0.8 & & & 2.1 & 0.8 & & & 2.1 & 0.8 \\
\hline \multicolumn{21}{|l|}{ Total measured hours } \\
\hline Total & & & 16.9 & 1.0 & & & 16.9 & 1.1 & & & 17.1 & 1.2 & & & 17.1 & 1.3 & & & 17.0 & 1.2 \\
\hline Work & & & 8.2 & 1.2 & & & 8.0 & 1.7 & & & 8.0 & 1.1 & & & 7.5 & 1.2 & & & 7.9 & 1.2 \\
\hline Leisure time & & & 8.7 & 1.3 & & & 8.9 & 1.4 & & & 9.1 & 1.6 & & & 9.6 & 1.8 & & & 9.1 & 1.6 \\
\hline \multicolumn{21}{|l|}{ Physical activity } \\
\hline OPA ( $\%$ of work hours) & & & 6.9 & 1.8 & & & 13.4 & 1.9 & & & 19.2 & 1.6 & & & 27.3 & 4.7 & & & 16.5 & 8.0 \\
\hline LTPA (\% of leisure-time) & & & 9.8 & 3.6 & & & 10.3 & 4.7 & & & 10.4 & 4.5 & & & 11.1 & 4.8 & & & 10.5 & 4.4 \\
\hline OPA (hours/day) & & & 0.6 & 0.2 & & & 1.1 & 0.2 & & & 1.5 & 0.2 & & & 2.0 & 0.4 & & & 1.3 & 0.6 \\
\hline LTPA (hours/day) & & & 0.8 & 0.3 & & & 0.9 & 0.4 & & & 1.0 & 0.4 & & & 1.1 & 0.5 & & & 0.9 & 0.4 \\
\hline
\end{tabular}


ing working hours consisted of about 150 minutes standing, 160 minutes sitting, 2 minutes lying, and 70 minutes of other movements. The remaining leisure time consisted of 105 minutes standing, 290 minutes sitting, 50 minutes lying, and 45 minutes of other movements.

\section{Associations of OPA and LTPA with sleep problems}

Table 2 shows the association for OPA, LTPA and total physical activity with insomnia symptoms. The continuous OPA variable (ie, OR per $10 \%$ difference) was positively associated with insomnia symptoms (OR $1.39,95 \%$ CI 1.03-1.89). Compared to workers with very low OPA, workers with low, medium, and high OPA had OR of 1.22 (95\% CI 0.58-2.56), 1.61 (95\% CI $0.78-3.34$ ), and 2.28 (95\% CI 1.10-4.75), respectively. The OR of insomnia symptoms associated with a $10 \%$ higher LTPA was 0.67 (95\% CI 0.38-1.19). Workers with low, medium, and high LTPA had OR ranging from 0.57 (95\% CI $0.30-1.09)$ to 0.71 (95\% CI $0.38-1.36$ ) compared to workers with very low LTPA. The OR of insomnia symptoms associated with a $10 \%$ higher total physical activity was 1.21 (95\% CI 0.72-2.01). Workers

Table 2. Association between occupational physical activity (OPA), leisure-time physical activity (LTPA), total physical activity (TPA) and insomnia symptoms. [OR=odds ratio; $\mathrm{Cl}=$ confidence interval.]

\begin{tabular}{|c|c|c|c|c|c|c|}
\hline \multirow[t]{2}{*}{ Physical activity } & \multirow{2}{*}{$\begin{array}{l}\text { Persons } \\
\text { (N) }\end{array}$} & \multirow{2}{*}{$\begin{array}{l}\text { Cases } \\
\text { (N) }\end{array}$} & \multicolumn{2}{|r|}{ Crude } & \multicolumn{2}{|c|}{ Multi-adjusted a } \\
\hline & & & OR & $95 \% \mathrm{Cl}$ & $O R$ & $95 \% \mathrm{Cl}$ \\
\hline \multicolumn{7}{|l|}{ OPA } \\
\hline Continuous ${ }^{b}$ & 650 & 92 & 1.39 & $1.06-1.82$ & 1.39 & $1.03-1.89$ \\
\hline \multicolumn{7}{|l|}{ Categorical c } \\
\hline Very low & 167 & 16 & 1.00 & Ref & 1.00 & Ref \\
\hline Low & 166 & 20 & 1.27 & $0.63-2.55$ & 1.22 & $0.58-2.56$ \\
\hline Medium & 162 & 27 & 1.87 & $0.96-3.62$ & 1.61 & $0.78-3.34$ \\
\hline High & 155 & 29 & 2.20 & $1.13-4.29$ & 2.28 & $1.10-4.75$ \\
\hline \multicolumn{7}{|l|}{ LTPA } \\
\hline Continuous b & 650 & 92 & 0.69 & $0.41-1.16$ & 0.67 & $0.38-1$. \\
\hline \multicolumn{7}{|l|}{ Categorical d } \\
\hline Very low & 164 & 28 & 1.00 & Ref & 1.00 & Ref \\
\hline Low & 163 & 21 & 0.74 & $0.40-1.36$ & 0.69 & $0.35-1.33$ \\
\hline Medium & 63 & 20 & 0.65 & $0.35-1.22$ & 0.57 & $0.30-1.09$ \\
\hline High & 30 & 23 & 0.78 & $0.42-1.43$ & 0.71 & $0.38-1.36$ \\
\hline \multicolumn{7}{|l|}{ TPA } \\
\hline Continuous ${ }^{b}$ & 650 & 92 & 1.24 & $0.79-1.94$ & 1.21 & $0.72-2.01$ \\
\hline \multicolumn{7}{|l|}{ Categorical e } \\
\hline Very low & 169 & 17 & 1.00 & $\operatorname{Re}$ & 1.00 & Ref \\
\hline Low & 161 & 25 & 1.64 & $0.85-3.18$ & 1.39 & $0.69-2.77$ \\
\hline Mediu & 164 & 25 & 1.61 & $0.83-3.11$ & 1.49 & $0.74-3.01$ \\
\hline High & 156 & 25 & 1.71 & $0.88-3.30$ & 1.62 & $0.79-3.32$ \\
\hline
\end{tabular}

a Multi-adjusted: age (continuous), sex, body mass index (continuous), pain (almost no pain, light pain, moderate pain, strong pain), alcohol consumption (low, medium, high), smoking (yes, no), work type (cleaning, manufacturing, transportation), shift work (fixed day work, night/ varying working hours, other), and use of anti-depressives (no, yes). ${ }^{\circ}$ Continuous OPA and LTPA variables (ie, OR per 10 minutes difference). ' Quartiles of OPA: very low (6-49 minutes), low (50-78 minutes), medium (79-102 minutes), high (103-235 minutes).

d Quartiles of LTPA: very low (7-38 minutes), low (39-52 minutes), medium (53-70 minutes), high (71-184 minutes).

${ }^{e}$ Quartiles of both OPA and LTPA: very low (24-101 min), low (102-128 minutes), medium (129-164 minutes), high (165-343 minutes). with low, medium, and high total physical activity had OR ranging from $1.39(95 \%$ CI $0.69-2.77)$ to $1.62(95 \%$ CI 0.79-3.32) compared to workers with very low total physical activity.

Table 3 shows the association for OPA, LTPA, and total physical activity with non-restorative sleep. The continuous LTPA variable (ie, OR per 10\% difference) was negatively associated with non-restorative sleep (OR 0.51, 95\% CI 0.28-0.92). Compared to workers with very low LTPA, workers with low, medium, and high LTPA had OR of 0.45 (95\% CI $0.24-0.85), 0.64$ (95\% CI $0.37-1.13)$, and 0.38 (95\% CI $0.20-0.71)$, respectively. The OR of non-restorative sleep associated with a $10 \%$ higher total physical activity was $0.57(95 \%$ CI 0.34-0.96). Workers with low, medium, and high total physical activity had OR ranging from 1.14 (95\% CI $0.63-2.05)$ to 0.69 (95\% CI 0.36-1.31) compared to workers with very low total physical activity.

\section{Joint association of OPA and LTPA with sleep problems}

Table 4 shows the joint association of OPA and LTPA with insomnia symptoms. We observed that people with

Table 3. Association between occupational physical activity (OPA), leisure-time physical activity (LTPA), total physical activity (TPA) and non-restorative sleep. [OR=odds ratio; $\mathrm{Cl}=$ confidence interval.]

\begin{tabular}{|c|c|c|c|c|c|c|}
\hline \multirow[t]{2}{*}{ Physical activity } & \multirow{2}{*}{$\begin{array}{l}\text { Persons } \\
\text { (N) }\end{array}$} & \multirow{2}{*}{$\begin{array}{l}\text { Cases } \\
\text { (N) }\end{array}$} & \multicolumn{2}{|r|}{ Crude } & \multicolumn{2}{|c|}{ Multi-adjusted a } \\
\hline & & & $\mathrm{OR}$ & $95 \% \mathrm{Cl}$ & $\mathrm{OR}$ & $95 \% \mathrm{Cl}$ \\
\hline \multicolumn{7}{|l|}{ OPA } \\
\hline Continuous ${ }^{b}$ & 650 & 112 & 0.97 & $0.76-1.23$ & 0.93 & $0.71-1.21$ \\
\hline \multicolumn{7}{|l|}{ Categorical c } \\
\hline Very low & 167 & 29 & 1.00 & Ref & 1.00 & Ref \\
\hline Low & 166 & 23 & 0.77 & $0.42-1.39$ & 0.78 & $0.40-1.51$ \\
\hline Medium & 162 & 36 & 1.36 & $0.79-2.35$ & 1.25 & $0.67-2.34$ \\
\hline High & 155 & 24 & 0.87 & $0.48-1.58$ & 0.85 & $0.45-1.59$ \\
\hline \multicolumn{7}{|l|}{ LTPA } \\
\hline Continuous ${ }^{b}$ & 650 & 112 & 0.53 & $0.31-0.91$ & 0.51 & $0.28-0.92$ \\
\hline \multicolumn{7}{|l|}{ Categorical d } \\
\hline Very low & 164 & 41 & 1.00 & Ref & 1.00 & Ref \\
\hline Low & 163 & 22 & 0.47 & $0.26-0.83$ & 0.45 & $0.24-0.85$ \\
\hline Mediur & 163 & 30 & 0.68 & $0.40-1.15$ & 0.64 & $0.37-1.13$ \\
\hline High & 160 & 19 & 0.40 & $0.22-0.73$ & 0.38 & $0.20-0.71$ \\
\hline \multicolumn{7}{|l|}{ TPA } \\
\hline Continuous ${ }^{b}$ & 650 & 112 & 0.63 & $0.40-1.00$ & 0.57 & $0.34-0.96$ \\
\hline \multicolumn{7}{|l|}{ Categorical e } \\
\hline Very low & 169 & 28 & 1.00 & $\mathrm{Re}$ & 1.00 & Ref \\
\hline Low & 161 & 33 & 1.30 & $0.74-2.27$ & 1.14 & $0.63-2.05$ \\
\hline Mediur & 164 & 31 & 1.17 & $0.67-2.06$ & 1.06 & $0.58-1.94$ \\
\hline High & 156 & 20 & 0.74 & $0.40-1.38$ & 0.69 & $0.36-1.31$ \\
\hline
\end{tabular}

a Multi-adjusted: age (continuous), sex, body mass index (continuous), pain (almost no pain, light pain, moderate pain, strong pain), alcohol consumption (low, medium, high), smoking (yes, no), work type (cleaning, manufacturing, transportation), shift work (fixed day work, night/ varying working hours, other), and use of anti-depressives (no, yes). ${ }^{b}$ Continuous OPA and LTPA variables (ie, OR per 10 minutes difference). ' Quartiles of OPA: very low (6-49 minutes), low (50-78 minutes), medium (79-102 minutes), high (103-235 minutes).

${ }^{d}$ Quartiles of LTPA: very low (7-38 minutes), low (39-52 minutes), medium (53-70 minutes), high (71-184 minutes).

e Quartiles of both OPA and LTPA: very low (24-101 min), low (102-128 minutes), medium (129-164 minutes), high (165-343 minutes). 
high OPA and low LTPA had an OR of 2.07 (95\% CI 1.01-4.24) for insomnia symptoms, compared to the reference group with low OPA and high LTPA, whereas people with high level of both OPA and LTPA had an OR of 1.47 (95\% CI 0.73-2.96). However, there was no evidence of interaction between OPA and LTPA on the association with insomnia symptoms on an additive (RERI) scale $(0.55,95 \%$ CI $-0.77-1.70)$

Table 5 shows the joint association of OPA and LTPA with non-restorative sleep. Compared to workers with high LTPA and low OPA, workers with the combination of low LTPA and high OPA had an OR of 1.79 (95\% CI 0.92-3.50). Furthermore, workers with the combination of low LTPA and low OPA had an OR of 1.68 (95\% CI 0.87-3.23) and workers with high LTPA and high OPA had an OR of 1.46 (95\% CI 0.75-2.84). However, there was no evidence of interaction between OPA and LTPA on the association with non-restorative sleep on an additive (RERI) scale (-0.35, 95\% CI -1.71-0.99).

\section{Sensitivity analysis}

The robustness of the results were assessed in three sensitivity analyses. Firstly, we used tertiles for OPA and LTPA. Compared to low OPA, high OPA had OR of insomnia symptoms of 1.83 (95\% CI 0.99-3.38). Compared to low LTPA, the medium and high LTPA had OR of insomnia symptoms of 0.51 (95\% CI $0.28-0.91)$ and 0.59 (95\% CI 0.34-1.02), respectively. Compared to low LTPA, high LTPA had an OR of non-restorative sleep of 0.45 (95\% CI 0.26-0.78). Secondly, when we excluded workers with night shifts, the association between OPA and insomnia symptoms became somewhat stronger; ie, compared to workers with very low OPA, workers with low, medium, and high OPA had OR of 0.95 (95\% CI 0.40-2.25), 1.93 (95\% CI 0.90-4.17), and 2.82 (95\%

Table 4. The joint association of occupational physical activity (OPA) and leisure-time physical activity (LTPA) with insomnia symptoms. [OR=odds ratio; $\mathrm{Cl}=$ confidence interval.]

\begin{tabular}{|c|c|c|c|c|c|c|c|}
\hline \multirow[b]{4}{*}{ OPA } & \multicolumn{7}{|c|}{ LTPA } \\
\hline & \multicolumn{4}{|c|}{ High a $^{a}$} & \multicolumn{3}{|c|}{ Low b } \\
\hline & $\begin{array}{l}\text { Persons } \\
\text { (N) }\end{array}$ & $\begin{array}{c}\text { Cases } \\
(\mathrm{N})\end{array}$ & $\mathrm{OR}^{\mathrm{C}}$ & $95 \% \mathrm{Cl}^{\mathrm{c}}$ & $\begin{array}{l}\text { Person } \\
\text { (N) }\end{array}$ & $\begin{array}{l}\text { Cases OR }{ }^{c} \\
(\mathrm{~N})\end{array}$ & $95 \% \mathrm{Cl}^{\mathrm{c}}$ \\
\hline & & & & & & & \\
\hline Low $^{d}$ & 152 & 16 & 1.00 & Ref & 181 & 1.05 & $0.51-2.18$ \\
\hline Highe & 171 & 27 & 1.47 & $0.73-2.96$ & 146 & $29 \quad 2.07$ & $1.01-4.24$ \\
\hline \multicolumn{8}{|c|}{$\begin{array}{l}\text { ' Multi-adjusted: age (continuous), sex, body mass index (continuous), } \\
\text { pain (almost no pain, light pain, moderate pain, strong pain), alcohol } \\
\text { consumption (low, medium, high), smoking (yes, no), sector (cleaning, } \\
\text { manufacturing, transportation), shift work (fixed day work, night/varying } \\
\text { working hours, other) and use of anti-depressives (no, yes). }\end{array}$} \\
\hline
\end{tabular}

CI 1.32-6.02), respectively. In the analyses of the joint association of OPA and LTPA with insomnia symptoms, the associations became stronger; ie, compared to workers with high LTPA and low OPA, workers with the combination of low LTPA and high OPA had an OR of 2.69 (95\% CI 1.17-6.16). The corresponding joint association with non-restorative sleep became slightly stronger compared to the original analyses; ie, compared to workers with high LTPA and low OPA, workers with the combination of low LTPA and high OPA had an OR of 2.11 (95\% CI 1.03-4.34). Furthermore, workers with the combination of low LTPA and low OPA had an OR of 1.83 (95\% CI 0.92-3.64) and workers with high LTPA and high OPA had an OR of 1.51 (95\% CI 0.75-3.06) compared to workers with high LTPA and low OPA. Finally, including working hours as a covariate in the model did not change the results.

\section{Discussion}

The main findings of this study were that workers who conducted higher levels of OPA reported more insomnia symptoms, while workers who conducted higher levels of LTPA reported less non-restorative sleep. The associations remained essentially unchanged when using continuous measures and tertiles of OPA and LTPA in our analyses. Interpretation of total physical activity should be made with care because of the opposite associations of OPA and LTPA with insomnia symptoms and non-restorative sleep. Although the analyses of joint associations showed no deviation from additive interaction between OPA and LTPA on insomnia symptoms and non-restorative sleep, workers with the combination of high OPA and low LTPA had more symptoms

Table 5. The joint association of occupational physical activity (OPA) and leisure-time physical activity (LTPA) with non-restorative sleep. [OR=0dds ratio; $\mathrm{Cl}=$ confidence interval.]

\begin{tabular}{|c|c|c|c|c|c|c|c|}
\hline & \multicolumn{7}{|c|}{ LTPA } \\
\hline & \multicolumn{4}{|c|}{ High a } & \multicolumn{3}{|c|}{ Low b } \\
\hline & $\begin{array}{l}\text { Persons } \\
\text { (N) }\end{array}$ & $\begin{array}{l}\text { Cases } \\
(\mathrm{N})\end{array}$ & $\mathrm{OR}^{\mathrm{c}}$ & $95 \% \mathrm{Cl}^{\mathrm{c}}$ & $\begin{array}{l}\text { Persons } \\
\text { (N) }\end{array}$ & $\begin{array}{l}\text { Cases OR }{ }^{c} \\
(\mathrm{~N})\end{array}$ & $95 \% \mathrm{Cl} c$ \\
\hline \multicolumn{8}{|l|}{ OPA } \\
\hline Low $^{d}$ & 152 & 16 & 1.00 & Ref & 181 & 1.68 & $0.87-3.23$ \\
\hline Highe & 171 & 27 & 1.46 & $0.75-2.84$ & 146 & $29 \quad 1.79$ & $0.92-3.50$ \\
\hline \multicolumn{8}{|c|}{ a High LTPA: "medium" and "high" LTPA. } \\
\hline \multicolumn{8}{|c|}{ b Low LTPA: "very low" and "low" LTPA. } \\
\hline \multirow{4}{*}{\multicolumn{8}{|c|}{$\begin{array}{l}\text { ' Multi-adjusted: age (continuous), sex, body mass index (continuous), } \\
\text { pain (almost no pain, light pain, moderate pain, strong pain), alcohol } \\
\text { consumption (low, medium, high), smoking (yes, no), sector (cleaning, } \\
\text { manufacturing, transportation), shift work (fixed day work, night/varying } \\
\text { working hours, other) and use of anti-depressives (no, yes). }\end{array}$}} \\
\hline & & & & & & & \\
\hline & & & & & & & \\
\hline & & & & & & & \\
\hline \multicolumn{8}{|c|}{ d Low OPA: "very low" and "low" OPA. } \\
\hline \multicolumn{8}{|c|}{ e High OPA: "medium" and "high" OPA. } \\
\hline
\end{tabular}


of insomnia compared to the combination of low OPA and high LTPA. Compared to low LTPA, the effect of high LTPA on insomnia symptoms did not diminish with increasing OPA. These results indicate that high LTPA may be beneficial even when combined with high levels of OPA. These findings of the joint effect of OPA and LTPA on insomnia symptoms and non-restorative sleep provide novel information about the interplay between domains of physical activity. Thus, these results indicate that the association between physical activity and sleep problems depends on the domain of physical activity.

Strengths of the current study include the objective measurements of OPA and LTPA, allowing precise exposure estimates and minimizing the potential bias and misclassification associated with self-reported measurements of physical activity $(28,29)$. Furthermore, the relatively large study population and the large variations in OPA and LTPA, along with available information on several covariates, represent apparent strengths of the study. Notably, the main results remained essentially unchanged after sensitivity analyses with night work and working hours being included as covariates, two known risk factors of sleep problems $(34,35)$. Moreover, few studies have investigated non-restorative sleep as a distinct symptom of insomnia. Some limitations should be considered in the interpretation of the results. Symptoms of insomnia were assessed by self-report rather than clinical diagnosis. The DPhacto study lacks information about insomnia symptoms beyond one month, and no information was available on repeated awakening during the night and daytime sleepiness. The two questions used in DPhacto, therefore, do not fulfil the DSM-V criteria for insomnia diagnosis (36). The sleep length from the actigraph accelerometers could not be determined in this study. Moreover, we investigated quartiles of physical activity within each domain, and other cut-points not based on the distributions of the specific study population should be investigated in studies with larger sample size. Furthermore, the cross-sectional design precludes the possibility to make causal inferences about the associations between OPA, LTPA, and sleep problems.

Some previous cross-sectional studies have investigated the association between OPA and indicators of poor or disturbed sleep $(26,27)$. Wennman and colleagues (27) showed that workers with high OPA are more likely to report reduced sleep duration and poor sleep quality. Likewise, a study by Åkerstedt and colleagues (26) showed that physically strenuous work such as repeated and monotonous movements, twisted work positon, breaking into sweat each day, shaking and vibration, and heavy lifting - was associated with disturbed sleep. In contrast, a prospective study with 2-years follow-up found that exposure to awkward work positions, heavy lifting or physical exertion above normal did not increase the risk of sleep problems (37).
These contrasting findings may relate to differences in study design and the assessment of OPA, LTPA, and sleep. A direct comparison with our results is difficult because of the focus on slightly different dimensions of sleep. Moreover, OPA and LTPA have in previous studies been assessed by self-reports. Importantly, it should be pointed out that previous studies mainly have focused on dimensions of OPA (eg, awkward work posture, lifting) other than those recorded in the current study. Although we based our assessment of OPA and LTPA on objective measurements, we did not assess physically strenuous work that include forceful work such as lifting, pushing, or pulling. Some differences between working hours and leisure time should be considered in the interpretation of the results. Compared to leisure time, we observed that working hours consisted of less lying and sitting and more total time on feet, such as standing. A notable difference between LTPA and OPA is that high LTPA included about 10 minutes with running and cycling, whereas high OPA included only about 1 minute with running or cycling. OPA is therefore performed with lower intensity, possibly over consecutive long working days and in combination with more time in a standing position, which is known not to promote cardiorespiratory fitness and health $(38,39)$. In contrast, LTPA may include periods with moderate-to-high intensity that are sufficient to improve cardiorespiratory fitness and health (40). Thus, the buffering effect of high LTPA among workers with high OPA could therefore be explained by the health- and fitness-promoting effect from high LTPA workers, making them more resilient for performing high OPA. The mechanisms behind the negative effects of OPA are still unknown (41), and further studies are needed to evaluate if some of the differences could be explained by a different time-pattern (bout length of continuous periods) of activity during work and leisure time. Moreover, different levels of decision authority and psychosocial stressors at work and during leisure time may also explain some of the contrasting health effects from the two domains of physical activity. For example, low influence at work in combination with high OPA could potentially lead to an overload response (42) resulting in increased heart rate, blood pressure, markers of inflammation (40), and elevated autonomous drive (43), which may cause insomnia symptoms (44).

Some evidence suggest that non-restorative sleep can occur without other insomnia symptoms (19), which underscores the importance of investigating non-restorative sleep as a distinct component of insomnia symptoms. In the present study, we found that LTPA was inversely associated with non-restorative sleep. This finding is supported by a previous study, showing that regular physical activity is associated with the feeling of being more rested in the morning, improved sleep quality, as well as less fragmented sleep (18). Noticeably, no clear 
independent association between OPA and non-restorative sleep was found. Thus, these results therefore indicate that physical activity may influence non-restorative sleep and insomnia symptoms differently.

Although the association between LTPA and sleep is not fully understood, regular exercise has been found to be associated with improved sleep parameters among good sleepers (45). Furthermore, LTPA can improve psychological health and well-being $(46,47)$, which in turn may reduce the risk of insomnia (48). Interestingly, both "low", "medium", and "high" LTPA were associated with reduced odds of insomnia and non-restorative sleep compared to the reference group with "very low" LTPA. Notably, the average time with LTPA was about 30 minutes/day in the "very low" and about 45 minutes/day in the "low" group. This result may indicate that LTPA benefits sleep when exceeding 45 minutes/day, with no further effect of increasing active time. To sum up, our results together with previous findings lend further support to the notion that regular LTPA improves sleep and should be recommended as a non-pharmacological treatment for disturbed sleep. Importantly, the current study indicates that OPA may have a negative effect on sleep. Thus, recommendations regarding physical activity and sleep should distinguish between LTPA and OPA.

Although our study provides novel information about OPA and sleep problems, future prospective studies are needed to disentangle the causal relation between domain-specific physical activity and risk of sleep problem. Moreover, future studies should aim at including objective assessments of all dimensions of OPA (eg, heavy lifting, static and repetitive movements, activity type, intensity, time pattern) and should also investigate the interplay between physical activity and psychosocial factors in both domains and risk of sleep problems. Finally, potential effect modification of sex and age on the association between physical activity and sleep problems ought to be investigated.

\section{Concluding remarks}

Our analyses showed that workers who conducted higher levels of OPA reported more insomnia symptoms, while workers who conducted higher levels of LTPA reported less non-restorative sleep. Although no statistical additive interaction was found, analyses of joint associations showed that high OPA and low LTPA were more strongly associated with insomnia symptoms compared to a combination of low OPA and high LTPA. The current study therefore calls attention to the domain-dependent association between physical activity and health-related outcomes, including sleep problems.

\section{Acknowledgement}

This work was supported by a grant to Eivind Schjelderup Skarpsno from the Liaison Committee between the Central Norway Regional Health Authority (RHA) and the Norwegian University of Science and Technology (NTNU). The National Research Centre for the Working Environment (NRCWE) financed the data collection of this study. The authors would like to thank the DPhacto research group and personnel who contributed to the data collection and analysis, especially Julie LagerstedOlsen, Dorte Ekner, Klaus Hansen, and Jørgen Skotte.

The authors declare no conflicts of interest.

\section{References}

1. LeBlanc M, Mérette C, Savard J, Ivers H, Baillargeon LM, Morin CM. Incidence and Risk Factors of Insomnia in a Population-Based Sample. Sleep. 2009;32(8):1027-37. https://doi.org/10.1093/sleep/32.8.1027.

2. Roth T. Insomnia: Definition, Prevalence, Etiology, and Consequences. J Clin Sleep Med. 2007;3(5):7-10.

3. Dew M, Hoch C, Buysse D, Monk T, Begley A, Houck P, et al. Healthy older adults' sleep predicts all-cause mortality at 4 to 19 years of follow-up. Psychosom Med. 2003;65(1):63-73. https://doi.org/10.1097/01.PSY.0000039756.23250.7C.

4. Vgontzas A, Liao D, Bixler E, Chrousos G, Vela-Bueno A. Insomnia with Objective Short Sleep Duration is Associated with a High Risk for Hypertension. Sleep.2009;32(4):491-7. https://doi.org/10.1093/sleep/32.4.491.

5. Sivertsen B, Krokstad S, Øverland S, Mykletun A. The epidemiology of insomnia: Associations with physical and mental health.: The HUNT-2 study. J Psychosom Res. 2009;67(2):109-16. https://doi.org/10.1016/j. jpsychores.2009.05.001.

6. Anothaisintawee T, Reutrakul S, Van Cauter E, Thakkinstian A. Sleep disturbances compared to traditional risk factors for diabetes development: systematic review and meta-analysis. Sleep Med Rev. 2016;30:11-24. https://doi.org/10.1016/j. smrv.2015.10.002.

7. Sofi F, Cesari F, Casini A, Macchi C, Abbate R, Gensini G. Insomnia and risk of cardiovascular disease: a meta-analysis. Eur J Cardiovasc Prev Rehabil. 2014;21(1):57-64. https://doi. org/10.1177/2047487312460020.

8. Mork PJ, Nilsen TI. Sleep problems and risk of fibromyalgia: longitudinal data on an adult female population in Norway. Arthritis Rheum. 2012;64(1):281-4. https://doi.org/10.1002/ art.33346.

9. Youngstedt SD, Kline CE. Epidemiology of exercise and sleep. Sleep Biol Rhythms. 2006;4(3):215-21. https://doi. org/10.1111/j.1479-8425.2006.00235.x.

10. Morgan K. Daytime activity and risk factors for late-life 
insomnia. J Sleep Res. 2003;12(3):231-8. https://doi. org/10.1046/j.1365-2869.2003.00355.x.

11. Ohida T, Kamal A, Uchiyama M, Kim K, Takemura S, Sone $\mathrm{T}$, et al. The influence of lifestyle and health status factors on sleep loss among the Japanese general population. Sleep. 2001;24(3):333-8. https://doi.org/10.1093/sleep/24.3.333.

12. Youngstedt SD, O'Connor PJ, Dishman RK. The effects of acute exercise on sleep: a quantitative synthesis. Sleep. 1997;20(3):203-14. https://doi.org/10.1093/sleep/20.3.203.

13. Driver HS, Taylor SR. Exercise and sleep. Sleep Med Rev. 2000;4(4):387-402. https://doi.org/10.1053/smrv.2000.0110.

14. Chennaoui M, Arnal PJ, Sauvet F, Leger D. Sleep and exercise: a reciprocal issue? Sleep Med Rev. 2015;20:59-72. https://doi. org/10.1016/j.smrv.2014.06.008.

15. Loprinzi PD, Cardinal BJ. Association between objectivelymeasured physical activity and sleep, NHANES 2005-2006. Ment Health Phys Act. 2011;4(2):65-9. https://doi. org/10.1016/j.mhpa.2011.08.001.

16. Flausino NH, Da Silva Prado JM, de Queiroz SS, Tufik $\mathrm{S}$, de Mello MT. Physical exercise performed before bedtime improves the sleep pattern of healthy young good sleepers. Psychophysiology. 2012;49(2):186-92. https://doi. org/10.1111/j.1469-8986.2011.01300.x.

17. Soltani M, Haytabakhsh MR, Najman JM, Williams GM, O'Callaghan MJ, Bor W, et al. Sleepless nights: the effect of socioeconomic status, physical activity, and lifestyle factors on sleep quality in a large cohort of Australian women. Arch Women Ment Health. 2012;15(4):237-47. https://doi. org/10.1007/s00737-012-0281-3.

18. King AC, Pruitt LA, Woo S, Castro CM, Ahn DK, Vitiello $\mathrm{MV}$, et al. Effects of moderate-intensity exercise on polysomnographic and subjective sleep quality in older adults with mild to moderate sleep complaints. J Gerontol A Biol Sci Med Sci. 2008;63(9):997-1004. https://doi.org/10.1093/ gerona/63.9.997.

19. Roth T, Zammit G, Lankford A, Mayleben D, Stern T, Pitman $\mathrm{V}$, et al. Nonrestorative sleep as a distinct component of insomnia. Sleep. 2010;33(4):449-58. https://doi.org/10.1093/ sleep/33.4.449.

20. Holtermann A, Burr H, Hansen JV, Krause N, Søgaard K, Mortensen OS. Occupational physical activity and mortality among Danish workers. Int Arch Occup Environ Health. 2012;85(3):305-10. https://doi.org/10.1007/s00420-0110668-x.

21. Holtermann A. Occupational and leisure-time physical activity and coronary heart disease. Occup Environ Med. 2015;72(9):615-6. https://doi.org/10.1136/ oemed-2015-102933.

22. Holtermann A, Marott JL, Gyntelberg F, Søgaard K, Mortensen OS, Prescott E, et al. Self-reported occupational physical activity and cardiorespiratory fitness: importance for cardiovascular disease and all-cause mortality. Scand J Work Environ Health. 2016;42(4):291-8. https://doi.org/10.5271/ sjweh.3563.

23. Harari G, Green MS, Zelber-Sagi S. Combined association of occupational and leisure-time physical activity with all-cause and coronary heart disease mortality among a cohort of men followed-up for 22 years. Occup Environ Med. 2015;72(9):617-24. https://doi.org/10.1136/ oemed-2014-102613.

24. Allesøe K, Søgaard K, Aadahl M, Boyle E, Holtermann A. Are hypertensive women at additional risk of ischaemic heart disease from physically demanding work? Eur J Prev Cardiol. 2016;23(10):1054-61. https://doi. org/10.1177/2047487316631681.

25. Holtermann A, Hansen JV, Burr H, Søgaard K, Sjøgaard G. The health paradox of occupational and leisure-time physical activity. Br J Sports Med. 2011;46(4):291-5. https://doi. org/10.1136/bjsm.2010.079582.

26. Åkerstedt T, Fredlund P, Gillberg M, Jansson B. Work load and work hours in relation to disturbed sleep and fatigue in a large representative sample. J Psychosom Res. 2002;53(1):585-8. https://doi.org/10.1016/S0022-3999(02)00447-6.

27. Wennman H, Kronholm E, Partonen T, Tolvanen A, Peltonen M, Vasankari T, et al. Physical activity and sleep profiles in Finnish men and women. BMC Public Health. 2014;14(1):8291. https://doi.org/10.1186/1471-2458-14-82.

28. Koch M, Lunde LK, Gjulem T, Knardahl S, Veiersted KB. Validity of questionnaire and representativeness of objective methods for measurements of mechanical exposures in construction and health care work. PLoS One. 2016;11(9):e0162881. https://doi.org/10.1371/journal. pone. 0162881 .

29. Gupta N, Heiden M, Mathiassen SE, Holtermann A. Prediction of objectively measured physical activity and sedentariness among blue-collar workers using survey questionnaires. Scand J Work Environ Health. 2016;42(3):237-45.

30. Jørgensen MB, Korshøj M, Lagersted-Olsen J, Villumsen M, Mortensen OS, Skotte J, et al. Physical activities at work and risk of musculoskeletal pain and its consequences: protocol for a study with objective field measures among blue-collar workers. BMC Musculoskelet Disord. 2013;14(1):213. https:// doi.org/10.1186/1471-2474-14-213.

31. Skotte J, Korshøj M, Kristiansen J, Hanisch C, Holtermann A. Detection of physical activity types using triaxial accelerometers. J Phys Act Health. 2014;11(1):76-84. https:// doi.org/10.1123/jpah.2011-0347.

32. Stemland I, Ingebrigtsen J, Christiansen CS, Jensen BR, Hanisch C, Skotte J, et al. Validity of the Acti4 method for detection of physical activity types in free-living settings: comparison with video analysis. Ergonomics. 2015;58(6):95365. https://doi.org/10.1080/00140139.2014.998724.

33. Andersson T, Alfredsson L, Källberg H, Zdravkovic S, Ahlbom A. Calculating measures of biological interaction. Eur J Epidemiol. 2005;20(7):575-9. https://doi.org/10.1007/ s10654-005-7835-x.

34. Akerstedt T, Nordin M, Alfredsson L, Westerholm P, Kecklund G. Sleep and sleepiness: impact of entering or leaving shiftwork--a prospective study. Chronobiol Int. 2010;27(5):987-96. https://doi.org/10.3109/07420528.2010 .489423 . 
35. Virtanen M, Ferrie JE, Gimeno D, Vahtera J, Elovainio M, Singh-Manoux A, et al. Long working hours and sleep disturbances: the Whitehall II prospective cohort study. Sleep. 2009;32(6):737-45. https://doi.org/10.1093/sleep/32.6.737.

36. American Psychiatric Association. Diagnostic and statistical manual of mental disorders. American Psychiatric Publishing: Washington DC; 2013 (fifth edition).

37. Åkerstedt $\mathrm{T}$, Garefelt J, Richter A, Westerlund H, Magnusson Hanson LL, Sverke M, et al. Work and sleep--a prospective study of psychosocial work factors, physical work factors, and work scheduling. Sleep. 2015;38(7):1129-36. https://doi. org/10.5665/sleep. 4828 .

38. Waters TR, Dick RB. Evidence of health risks associated with prolonged standing at work and intervention effectiveness. Rehabil Nurs. 2015;40(3):148-65. https://doi.org/10.1002/ rnj.166.

39. Coenen P, Parry S, Willenberg L, Shi JW, Romero L, Blackwood DM, et al. Associations of prolonged standing with musculoskeletal symptoms- a systematic review of laboratory studies. Gait Posture. 2017;58:310-18. https://doi. org/10.1016/j.gaitpost.2017.08.024.

40. Warburton DER, Nicol CW, Bredin SSD. Health benefits of physical activity: the evidence. CMAJ. 2006;174(6):801-9. https://doi.org/10.1503/cmaj.051351.

41. Holtermann A, Krause N, van der Beek AJ, Straker L. The physical activity paradox: six reasons why occupational physical activity (OPA) does not confer the cardiovascular health benefits that leisure time physical activity does. $\mathrm{Br}$ J Sports Med. 2017. [E-pub ahead of print] https://doi. org/10.1136/bjsports-2017-097965.
42. Sluiter JK, Frings-Dresen MH, Meijman TF, van der Beek AJ. Reactivity and recovery from different types of work measured by catecholamines and cortisol: a systematic literature overview. Occup Environ Med. 2000;57(5):298-315. https:// doi.org/10.1136/oem.57.5.298.

43. Hallman DM, Birk Jørgensen M, Holtermann A. On the health paradox of occupational and leisure-time physical activity using objective measurements: Effects on autonomic imbalance. PloS One. 2017;12(5):e0177042. https://doi. org/10.1371/journal.pone.0177042.

44. Han KS, Kim L, Shim I. Stress and Sleep Disorder. Exp Neurobiol. 2012;21(4):141-50. https://doi.org/10.5607/ en.2012.21.4.141.

45. Kubitz KA, Landers DM, Petruzzello SJ, Han M. The effects of acute and chronic exercise on sleep. A metaanalytic review. Sports Med. 1996;21(4):277-91. https://doi. org/10.2165/00007256-199621040-00004.

46. Park S. Associations of physical activity with sleep satisfaction, perceived stress, and problematic internet use in Korean adolescents. BMC Public Health. 2014;14(1):1143. https://doi.org/10.1186/1471-2458-14-1143.

47. Teychenne M, Costigan SA, Parker K. The association between sedentary behaviour and risk of anxiety: a systematic review. BMC Public Health. 2015;15(1):513. https://doi. org/10.1186/s12889-015-1843-x.

48. Smagula SF, Reynolds CF 3rd, Ancoli-Israel S, Barrett-Connor E, Dam TT, Hughes-Austin JM, et al. Sleep architecture and mental health among community-dwelling older men. J Gerontol B Psychol Sci Soc Sci. 2015;70(5):673-81. https:// doi.org/10.1093/geronb/gbt125.

Received for publication: 2 June 2017 